\title{
The First Direct Distance and Luminosity Determination for a Self-Luminous Giant Exoplanet: The Trigonometric Parallax to 2MASSW J1207334-393254Ab
}

\author{
B.A. Biller ${ }^{1} \&$ L.M. Close $^{1}$ \\ bbiller@as.arizona.edu \\ ${ }^{1}$ Steward Observatory, University of Arizona, Tucson, AZ 85721
}

\begin{abstract}
We present the first trigonometric parallax and distance for a young planetary mass object. A likely TW Hya cluster member, 2MASSW J1207334-393254Ab (hereafter 2M1207Ab) is an M8 brown dwarf with a mid to late L type planetary mass companion. Recent observations of spectral variability have uncovered clear signs of disk accretion and outflow, constraining the age of the system to $<10$ Myr. Because of its late spectral type and the clearly youthful nature of the system, 2M1207b is very likely a planetary mass object. We have measured the first accurate distance and luminosity for a self-luminous planetary mass object. Our parallax measurements are accurate to $<2$ mas $(1 \sigma)$ for 2M1207Ab. With 11 total epochs of data taken from January 2006 through April 2007 (475 images for $2 \mathrm{M} 1207 \mathrm{Ab})$, we determine a distance of $58.8 \pm 7.0 \mathrm{pc}\left(17.0_{-1.8}^{+2.3}\right.$ mas, $\left.1.28 \sigma\right)$ to $2 \mathrm{M} 1207 \mathrm{Ab}$ and a calculated luminosity of $0.68-2.2 \times 10^{-5} \mathrm{~L}_{\odot}$ for $2 \mathrm{M} 1207 \mathrm{~b}$. Hence $2 \mathrm{M} 1207 \mathrm{Ab}$ is a clear member of the TW Hya cluster in terms of its distance, proper motions, and youthful nature. However, as previously noted by Mohanty and co-workers, 2M1207b's luminosity appears low compared to its temperature according to evolutionary models.
\end{abstract}

Subject headings: binaries: brown dwarfs, extrasolar planets

\section{Introduction}

A very likely member of the $\sim 8$ Myr TW Hydrae association (at distances of 35-70 pc, Mohanty et al. 2003, Mamajek 2005), 2MASSW J1207334-393254A (hereafter 2M1207A) is a young M8 brown dwarf with a late L companion (2M1207b, Chauvin et al. 2004, spectral type from Mohanty et al. 2007). Recent observations of spectral variability (Scholz and 
Jayawardhana 2006) have revealed accretion and jet-like features, constraining the age of the system to $<10 \mathrm{Myr}$; with such a young age and low temperature, 2M1207b is very likely a planetary mass object.

Interestingly, previous distance estimates for this object have produced somewhat conflicting results. Using the measured $\mathrm{K}$ band magnitudes and extrapolating a $\mathrm{K}$ band absolute magnitude for a 8 Myr brown dwarf (from trends in Song et al. 2003), Chauvin et al. (2004) estimated a photometric distance of $\sim 70$ pc at the outer edge of TW Hya from their extrapolated distance modulus. At this age and distance, the models of Baraffe et al. (2001) predict that the M8 dwarf should have a mass of $25 \mathrm{M}_{J u p}$ and the companion should have a mass of $\sim 5 \mathrm{M}_{\text {Jup }}$. Mamajek (2005) estimates a theoretical moving cluster distance to $2 \mathrm{M} 1207$ of $53 \pm 6 \mathrm{pc}$ and infers masses for $2 \mathrm{M} 1207 \mathrm{~A}$ and $\mathrm{b}$ (with an age of $8 \mathrm{Myr}$ ) of $\sim 21 \mathrm{M}_{\text {Jup }}$ and $\sim 3-4$ $\mathrm{M}_{\text {Jup }}$ respectively. From precision HST proper motions, Song et al. (2006) also estimate a similar moving cluster distance $-59 \pm 7 \mathrm{pc}$, and hence similar masses. However, using these closer distance estimates, 2M1207A becomes underluminous and falls nearer the locus of the (120 Myr) Pleiades on a color-magnitude diagram (Fig. 1) - which is inconsistent with an object age of $<10$ Myr at $\sim 50$ pc. Recently, Mamajek and Meyer (2007) have revised the estimated theoretical moving cluster distance to $2 \mathrm{M} 1207$ to $66 \pm 5$ pc. Thus, a direct distance measurement via parallax would help clarify this situation and additionally would constrain a number of important properties for this object. Since the youth ( $<10 \mathrm{Myr}$ ) and hence low mass nature $\left(\mathrm{M}<13 \mathrm{M}_{\text {Jup }}\right)$ of $2 \mathrm{M} 1207 \mathrm{~b}$ has recently been confirmed, we have also measured the first accurate luminosity for a self-luminous planetary mass object.

\section{Observations and Data Reduction}

We have acquired 11 epochs of data stretching from January 2006 to April 2007 with ANDICAM at the SMARTS $1.3 \mathrm{~m}$ telescope on Cerro Tololo. Both 2M1207 and the standard object LHS 2397a (an M8 dwarf with an L7.5 brown dwarf companion with a well known parallax of $62.3 \pm 4.0$ mas - Tinney (1996)) were observed for 40 minutes over transit. Observations were repeated over multiple nights bracketing the new moon each month. Over the entire observing period, $475 \mathrm{I}$ band data frames with per frame exposure time of $300 \mathrm{~s}$ were acquired for 2M 1207 and 491 I band data frames were acquired for LHS 2397a.

The M8 primary of 2M1207 has I=15.8 (Scholz et al. 2005) so parallax can be determined in the visible. The L5-9.5 secondary of 2M1207 has $\mathrm{K}=16.9$ (Chauvin et al. 2004), is even fainter in the visible $(\mathrm{I}>19)$, and lies within 0.8 " of its primary, thus it is essentially invisible to the $1.3 \mathrm{~m}$ in the optical (and is not apparent in our images). Its presence does not affect our attainable astrometric precision. LHS 2397a is comparably bright as 2M1207 and was 
chosen as a standard object due to the fact that both are late $\mathrm{M}$ dwarf + mid to late L dwarf binaries (Freed et al. 2003). Our measurement of the parallax of LHS 2397a (previously found to be $62.3 \pm 4.0$ mas, Tinney (1996)) serves as a test of our observational and data reduction procedures. Each target object was always placed at 82 pix E and 148 pix $\mathrm{N}$ of the center of the chip at pixel $(330,660)$. A number of bright stars lie right outside the LHS 2397a field; we chose to place our target objects in the upper left quadrant of the chip as opposed to the center of the chip in order to keep these bright stars and their saturation bleeds off of the CCD.

A dedicated parallax data analysis pipeline was used to reduce these data. This pipeline aligns each data frame to a master frame, removes cosmic rays, and performs PSF fitting photometry for 139 stars per frame using the DAOphot allstar task (Stetson 1987). The ANDICAM optical detector is a Fairchild $4472048 \times 2048$ CCD and was used in $2 \times 2$ binning mode yielding a nominal platescale of $0.369 \mathrm{arcsec} /$ pixel (ANDICAM website). Five bright stars in different parts of the field were used to calibrate $\mathrm{x}$ and $\mathrm{y}$ plate scale changes on the CCD. The separations between the 5 platescale calibration stars were measured for each data frame and then normalized to the average value over all frames. These normalized values were used as frame by frame platescale corrections. We found platescale variations of less than $0.06 \%$ over the $\sim 1.5$ year time baseline of our observations"

The position of 2M1207 and LHS 2397a in RA and DEC as a function of time is shown in Figs. 3. Many reference stars as bright or brighter than the target are available in both the 2M1207 and LHS 2397a fields; thus, we have a good distribution of baselines around both objects from which to calculate parallax. The position of 2M1207 and LHS 2397a were measured relative to 40 and 15 reference stars respectively.

To correct for a slightly variable $\mathrm{x}$ and $\mathrm{y}$ "pincushion" distortion (first derivative of the $\mathrm{x}$ and $\mathrm{y}$ platescale) on the chip, we measured the apparent motion on the sky for the 5 stars nearest 2M1207 on the chip. These stars are faint and likely significantly behind 2M1207 and thus presumed background so the motion observed for these should calibrate the local distortion of the chip near our target. Two of these stars showed apparent space motions of their own and were thus discarded. The motions from the remaining three stars were then averaged together to create a first derivative platescale correction curve. This correction curve was then subtracted from the measured parallax curve for 2M1207. A similar correction was also performed for the LHS 2397a data.

To determine the trigonometric parallax to 2M1207 and LHS 2397a, we fit (least squares fit) our measured parallax data to a parallax and proper motion model for each dataset. Precise HST proper motions for 2M1207 of $\mu_{\alpha}=-60.2 \pm 4.9 \mathrm{mas} / \mathrm{yr}$ and $\mu_{\delta}=-25.0 \pm 4.9 \mathrm{mas} / \mathrm{yr}$ were adopted from Song et al. (2006). Proper motions for LHS 2397a of $\mu_{\alpha}=-508.0 \pm 20.0$ 
mas/yr and $\mu_{\delta}=-80.0 \pm 20.0 \mathrm{mas} / \mathrm{yr}$ were adopted from Salim \& Gould (2003). Fits were performed both holding proper motion fixed and also fitting to proper motion; for both 2M1207 and LHS 2397a we retrieve the published proper motions to within the published errors. We find that the error in published proper motion is negligible compared to per frame measurement errors. Error in each nightly parallax measurement was calculated from the rms of measurements taken over that night. Measurements from late 2006 (2006.9 - 2007.1 epoch) were discarded since these data were taken off transit and thus suffer considerably from Differential Color Refraction (DCR, see, e.g. Dahn et al. 2002).

In order to estimate the error in our trigonometric parallax measurement, a Monte-Carlo ensemble of 10000 datasets was simulated by multiplying our measured position errors by a random number distribution pulled from a Gaussian distribution, then adding that random error to the measured position. An error-free trigonometric parallax model was then fitted and chi-square minimized to each of these simulated observations. The adopted distance is the mean of this 10000 simulated observation distribution of fits and the adopted error is the $1.28 \times$ the standard deviation of this distribution, corresponding to an $80 \%$ confidence interval. Histograms of the distributions of observation fits are presented in Fig. 2.

We estimate a correction from relative to absolute parallax of $1.2 \pm 0.9$ mas, based on I band photometry of 40 reference stars for 2M1207 and 15 reference stars for LHS 2397a. This correction was obtained by adopting an average spectral type of M0 for our relatively faint field reference stars ( $\mathrm{I}=15-18 \mathrm{mag}$ ), calculating photometric parallaxes for each reference star and then employing the median photometric parallax of the reference stars as the estimated correction.

\section{Results and Discussion}

For 2M1207Ab, we acquired a best fit relative parallax of $15.8_{-1.6}^{+2.1}$ mas, corresponding to an absolute parallax of $17.0_{-1.8}^{+2.3}$ mas and a best fit distance of $58.8 \pm 7.0 \mathrm{pc}$ (all $1.28 \sigma$ errors). For our standard LHS 2397a, we acquired a best fit relative parallax of $66.7_{-4.6}^{+5.2}$ mas, corresponding to an absolute parallax of $67.9_{-4.7}^{+5.3}$ mas (similar to the previous result of $62.6 \pm 4.0$ mas, Tinney 1996) and a best fit distance of $14.7 \pm 1.0$ pc. Fewer reference stars were available for the LHS 2397a standard object than for 2M1207, leading to a lower precision result. Parallax results are presented in Table 1. Absolute magnitudes derived using our measured distance are presented in Table 2.

Adopting an apparent $\mathrm{J}$ magnitude of $13.00 \pm 0.03$ (Mohanty et al. 2007) and $\mathrm{BC}_{J}=2.0$ for a M8 (Dahn et al. 2002), we estimate a total luminosity for $2 \mathrm{M} 1207 \mathrm{~A}$ of $2.7 \times 10^{-3} \mathrm{~L}_{\odot}$. 
Adopting an apparent $\mathrm{J}$ magnitude of $20.00 \pm 0.02$ (Mohanty et al. 2007) and $\mathrm{BC}_{J}=1.5$ for a late L dwarf (Dahn et al. 2002), we estimate a total luminosity for $2 \mathrm{M} 1207 \mathrm{~b}$ of $6.8 \times 10^{-6}$ $\mathrm{L}_{\odot}$. We repeated this calculation in the Ks band: adopting $\mathrm{m}_{K s}=11.95 \pm 0.03$ (Chauvin et al. 2004) and $\mathrm{BC}_{K}=3.2$ (Golimowski et al. 2004) for $2 \mathrm{M} 1207 \mathrm{~A}$ and converting $\mathrm{m}_{K s}$ to $\mathrm{m}_{K}$ using the transformations from Carpenter (2001), we estimate a total luminosity of $2.4 \times 10^{-3} \mathrm{~L}_{\odot}$, consistent with the $\mathrm{J}$ band estimate. However, adopting $\mathrm{m}_{K s}=16.93 \pm 0.11$ (Chauvin et al. 2004) and $\mathrm{BC}_{K}=3.3-3.4$ (Golimowski et al. 2004) for $2 \mathrm{M} 1207 \mathrm{~b}$ and converting $\mathrm{m}_{K s}$ to $\mathrm{m}_{K}$ using the transformations from Stephens \& Leggett (2004), we estimate a total luminosity of $2.0-2.2 \times 10^{-5} \mathrm{~L}_{\odot}-3 \times$ brighter than the $\mathrm{J}$ band estimate. The culprit here is likely the bolometric corrections used - which, while appropriate for older field objects are not entirely appropriate for this very young, very cool object. Accordingly, we do not assign error bars to our luminosity estimates because of the uncertainties in the bolometric corrections. $2 \mathrm{M} 1207 \mathrm{~b}$ is somewhat redder $(\mathrm{J}-\mathrm{K})$ than field objects of the same spectral types. Thus, $\mathrm{BC}_{K}$ is especially suspect, since the $\mathrm{K}$ band flux of $2 \mathrm{M} 1207 \mathrm{~b}$ represents a larger portion of its total bolometric flux than is true for comparable spectral type field objects. Estimated luminosities are presented in Table 2.

We estimated mass and effective temperatures for 2M1207Ab using only our derived absolute magnitudes and the DUSTY models of Chabrier et al. (2000) and Baraffe et al. (2001). Adopting an isochronal age for the TWA Hydra cluster of $\sim 8_{-3}^{+4} \mathrm{Myr}$ (Song et al. 2003; Zuckerman \& Song 2004; Chauvin et al. 2004), we compared the absolute J, H, $\mathrm{K}_{s}$, and $\mathrm{L}^{\prime}$ colors (after converting from 2MASS to CIT magnitudes) to the 5 and $10 \mathrm{Myr}$ isochrones. 2M1207A is consistent in color and luminosity with the 20-30 $\mathrm{M}_{\text {Jup }}$ models. $2 \mathrm{M} 1207 \mathrm{~b}$ is roughly consistent in luminosity with the $3-7 \mathrm{M}_{J u p}$ models (early $\mathrm{T}$ spectral types) - however, it possesses much redder colors than these models, consistent with midto-late $\mathrm{L}$ dwarfs (6-10 $\mathrm{M}_{\text {Jup }}$ models). This color/luminosity mismatch has been previously noted by Mohanty et al. (2007) among others - 2M1207b possesses a mid-late L spectrum and colors, but is underluminous for its age, possessing the luminosity expected of an early $\mathrm{T}$, yet no methane absorption is observed. From spectral fitting, $\mathrm{T}_{\text {eff }} \sim 1600 \mathrm{~K}$ for $2 \mathrm{M} 1207 \mathrm{~b}$ (Mohanty et al. 2007), yet we derive an incorrect model $\mathrm{T}_{\text {eff }}$ of only 1260-1430 K. Put in other words, according to the models $2 \mathrm{M} 1207 \mathrm{~b}$ is $10 \times$ too faint for its spectral type and age. A comparison of model to observed properties is presented in Table 3.

At a distance of $\sim 50 \mathrm{pc}$, both $2 \mathrm{M} 1207 \mathrm{~A}$ and $\mathrm{b}$ are somewhat underluminous for their respective spectral types (see Fig. 1). Increasing the distance to $59 \mathrm{pc}$ and adopting $\mathrm{T}_{\text {eff }}=2550 \pm 150$ pc (Mohanty et al. 2007) and age $=5-10 \mathrm{Myr}$ solves the underluminosity issue for 2M1207A, which becomes consistent with the $30 \mathrm{M}_{\text {Jup }}$, $10 \mathrm{Myr}$ DUSTY models to within 0.2 mag. However, adopting $\mathrm{T}_{e f f}=1600 \pm 100 \mathrm{~K}$ (Mohanty et al. 2007) and age $=5-10 \mathrm{Myr}$, at a distance of $59 \mathrm{pc}, 2 \mathrm{M} 1207 \mathrm{~b}$ is still underluminous compared to the models by $2-3$ mag in $\mathrm{JHK}_{s} \mathrm{~L}^{\prime}$. 
Thus, our increase in distance does not resolve the issue of 2M1207b's underluminosity.

A number of reasons for the lower than expected luminosity of $2 \mathrm{M} 1207 \mathrm{~b}$ have been suggested. Mohanty et al. 2007 suggest that an edge-on disk around 2M1207b produces $\sim 2.5$ mag of gray extinction (over $\mathrm{JHK}_{s} \mathrm{~L}^{\prime}$ ) and hence, the observed low luminosity. Mamajek and Meyer (2007) suggest that 2M1207b may be a hot protoplanet collision remnant. Additionally, while equally unlikely, 2M1207b may not be coeval with 2M1207A and may indeed be an older, smaller hence less luminous captured L dwarf (or rather, 2M1207A was captured by an old $\sim 60 \mathrm{M}_{\text {Jup }} \mathrm{L}$ dwarf). Indeed, the measured colors, absolute magnitudes $\left(\mathrm{JHK}_{s} \mathrm{~L}^{\prime}\right)$, and luminosity of $2 \mathrm{M} 1207 \mathrm{~b}$ are consistent with that of a $10 \mathrm{Gyr} 67 \mathrm{M}_{\text {Jup }}$ object with $\mathrm{L}$ spectral type and $\mathrm{T}_{e f f}=1500$.

The culprit could simply be the initial conditions of the evolutionary models used to derive physical properties. Marley et al. (2007) have noted that the "hot-start" evolutionary models for planets and brown dwarfs such as those from both the Lyon and Tucson groups (Baraffe et al. 2003, Burrows et al. 2003) possess very high initial entropies and predict considerably brighter luminosities for young high mass planets than models which start with lower entropy initial conditions (which may be more appropriate for planets which form via core accretion). For 4-10 $\mathrm{M}_{\text {Jup }}$ objects, the Marley et al. (2007) models converge with the standard evolutionary models by $100 \mathrm{Myr}$. While 2M1207b most likely formed via fragmentation from a cloud core rather than core accretion or gravitational collapse within 2M1207A's small disk, the initial entropy conditions of its formation might have been considerably lower than those utilized by standard evolutionary models, producing a lower luminosity for each spectral type than expected at very young ages. In particular, inside a binary system, the initial entropy conditions may have been different, presumably lower, for a forming $8 \mathrm{M}_{\text {Jup }}$ object interacting with a $30 \mathrm{M}_{\text {Jup }}$ "primary" than for a $8 \mathrm{M}_{\text {Jup }}$ object forming individually. Mohanty et al. (2007) claim that the models are not the culprit for 2M1207b's underluminous nature, comparing it with the young, low mass brown dwarf AB Pic B, whose colors, $\mathrm{T}_{\text {eff }}$, and luminosity agree well with the models. However, AB Pic is somewhat older than 2M1207Ab (30 Myr vs. $<10 \mathrm{Myr}$ ) and has a much wider separation between components (250 AU vs. $50 \mathrm{AU}$ ), so it may have already converged to the standard evolutionary model tracks.

\section{Conclusions}

We measured a distance of $58.8 \pm 7.0 \mathrm{pc}\left(17.0_{-1.8}^{+2.3}\right.$ mas $)$ to $2 \mathrm{M} 1207 \mathrm{Ab}$ and a luminosity of $0.68-2.2 \times 10^{-5} \mathrm{~L}_{\odot}$ for $2 \mathrm{M} 1207 \mathrm{~b}$, making $2 \mathrm{M} 1207 \mathrm{Ab}$ a clear member of the TW Hya cluster. While 2M1207A now agrees well with evolutionary models, 2M1207b is still 2-3 mag 
underluminous in $\mathrm{JHK}_{s} \mathrm{~L}^{\prime}$ for its suggested $\mathrm{T}_{\text {eff }}$ of $1600 \pm 100 \mathrm{~K}$ (Mohanty et al. 2007) and age of $\sim 8_{-3}^{+4}$ Myr (for the TW Hya cluster, Song et al. 2003).

This publication is based on observations made with the NOAO $1.3 \mathrm{~m}$ telescope operated by the SMARTS consortium. We acknowledge the excellent telescope queue support through the SMARTS consortium and would especially like to thank Jenica Nelan, Charles Bailyn, Juan Espinoza, David Gonzalez, and Alberto Pasten. We thank Matt Kenworthy for the suggestion of the Monte Carlo observation fits and Eric Mamajek for useful suggestions. BAB was supported by the NASA GSRP grant NNG04GN95H and NASA Origins grant NNG05GL71G. LMC is supported by an NSF CAREER award and the NASA Origins of the Solar System program.

\section{REFERENCES}

Baraffe, I., Chabrier, G., Allard, F., Hauschildt, P.H. 2001, A\&A, 382, 563

Baraffe, I., Chabrier, G., Barman, T.S., Allard, F., \& Hauschildt, P.H. 2003, A\&A 402, 701

Burrows, A., Sudarsky, D. \& Lunine, J. 2003, ApJ 596, 587

Carpenter, , J. M. 2001, AJ, 121, 2851

Chabrier, G., Baraffe, I., Allard, F., Hauschildt, P.H. 2000, ApJ,542, 464

Chauvin, G., Lagrange, A.-M., Dumas, C., Zuckerman, B., Mouillet, D., Song, I., Beuzit, J.-L., \& Lowrance, P. 2004, A\&A, 425, L29

Dahn, C. et al. 2002, AJ, 124, 1170

Freed, M., Close, L. M., \& Siegler, N. 2003, ApJ, 584, 453

Golimowski, D., et al. 2004, AJ, 127, 3516

Mamajek, E. 2005, ApJ, 634, 1385

Mamajek \& Meyer, in press.

Marley, M. S., Fortney, J. J., Hubickyj, O., Bodenheimer, P., \& Lissauer, J. J. 2007, ApJ, 655,541

Martín, E. L., Brandner, W., Bouvier, J., Luhman, K. L., Stauffer, J., Basri, G., Zapatero Osorio, M. R., \& Barrado y Navascués, D. 2000, ApJ, 543, 299 
Mohanty, S., Jayawardhana, R., \& Barrado y Navascués, D. 2003, ApJ, 593, L109

Mohanty, S., Jayawardhana, R., Huélamo, N., \& Mamajek, E. 2007, ApJ, 657, 1064

Salim, S., \& Gould, A. 2003, ApJ, 582, 1011

Scholz, A., Jayawardhana, R., \& Brandeker, A. 2005, ApJ, 629, L41

Scholz, A., \& Jayawardhana, R. 2006, ApJ, 638, 1056

Song, I., Zuckerman, B., \& Bessell, M. 2003, ApJ, 599, 342

Song, I., Schneider, G., Zuckerman, B., Farihi, J., Becklin, E. E., Bessell, M. S., Lowrance, P., \& Macintosh, B. A. 2006, ApJ, 652, 724

Stephens, D., \& Leggett, S. 2004, PASP, 116, 9

Stetson, P. B. 1987, PASP, 99, 191

Tinney, C. G. 1996, MNRAS, 281, 644

Zuckerman, B., \& Song, I. 2004, ARA\&A, 42, 685 
Table 1. Parallax Results

\begin{tabular}{cccccc}
\hline \hline Target & RA & DEC & $\pi$ (relative) & $\pi$ (absolute) & Distance \\
\hline 2M1207Ab & 120733.4 & -393254.0 & $15.8_{-1.6}^{+2.1}$ mas & $17.0_{-1.8}^{+2.3}$ mas & $58.8 \pm 7.0 \mathrm{pc}$ \\
LHS 2397a & 112149.2 & -131308.4 & $66.7_{-4.2}^{+4.8}$ mas & $67.9_{-4.7}^{+5.3}$ mas & $14.7 \pm 1.0 \mathrm{pc}$ \\
\hline
\end{tabular}

Table 2. Properties of $2 \mathrm{M} 1207 \mathrm{Ab}$

\begin{tabular}{cccccc}
\hline \hline Target & $\mathrm{M}_{J}^{\mathrm{a}}$ & $\mathrm{M}_{H}^{\mathrm{b}}$ & $\mathrm{M}_{K s} \mathrm{~b}^{\mathrm{b}}$ & $\mathrm{M}_{L}^{\mathrm{b}}$ & Luminosity $\left(\mathrm{L}_{\text {obs }}\right)$ \\
\hline 2M1207A & $9.15_{-0.24}^{+0.28}$ & $8.54_{-0.24}^{+0.28}$ & $8.10_{-0.24}^{+0.28}$ & $7.53_{-0.24}^{+0.29}$ & $2.4-2.7 \times 10^{-3}$ \\
2M1207b & $16.15_{-0.31}^{+0.34}$ & $14.24_{-0.32}^{+0.35}$ & $13.08_{-0.26}^{+0.30}$ & $11.43_{-0.28}^{+0.31}$ & $0.68-2.2 \times 10^{-5}$ \\
\hline
\end{tabular}

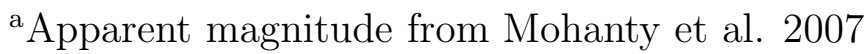

${ }^{\mathrm{b}}$ Apparent magnitude from Chauvin et al. 2004

Table 3. Comparison of Measured to Model Properties (5-10 Myr DUSTY models)

\begin{tabular}{ccccc}
\hline \hline Target & $\mathrm{T}_{\text {effobs }}{ }^{\mathrm{a}}$ & $\mathrm{T}_{\text {effmodel }}{ }^{\mathrm{b}}$ & Mass from $\mathrm{L}_{\text {obs }}$ & Mass from $\mathrm{T}_{\text {effobs }}{ }^{\mathrm{a}}$ \\
\hline 2M1207A & $2550 \pm 150 \mathrm{~K}$ & $2500-2700 \mathrm{~K}$ & $20-30 \mathrm{M}_{\text {Jup }}$ & $20-30 \mathrm{M}_{\text {Jup }}$ \\
2M1207b & $1600 \pm 100 \mathrm{~K}$ & $1260-1430 \mathrm{~K}$ & $3-7 \mathrm{M}_{\text {Jup }}$ & $6-10 \mathrm{M}_{\text {Jup }}$ \\
\hline
\end{tabular}

${ }^{a}$ from Mohanty et al. 2007

${ }^{\mathrm{b}} \mathrm{T}_{\text {eff }}$ derived from age and luminosity $\left(\mathrm{L}_{o b s}\right)$. 


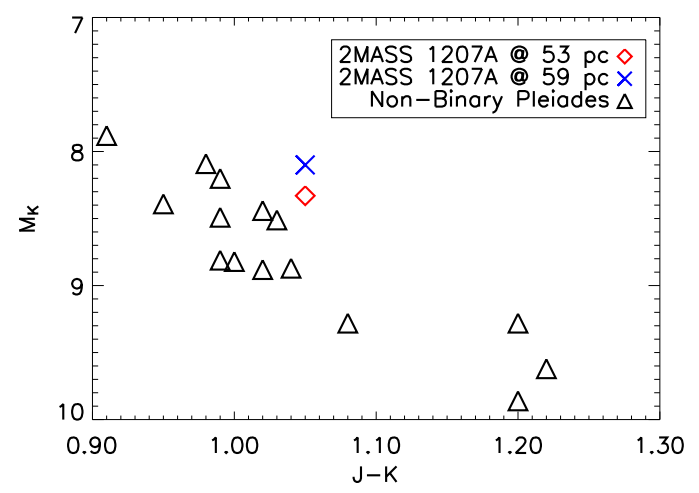

Fig. 1. $-\mathrm{M}_{K}$ vs. J-K HR diagram. Previous distance estimates for 2M1207A have led to a number of inconsistencies. J and K magnitudes are taken from the 2MASS survey. Triangle points are non-binary Pleiades members from Martín et al. (2000). At the Mamajek (2005) moving cluster distance of 53 pc, 2M1207A falls very close to the locus of the Pleiades (120 Myr). This is inconsistent with the age of $<10 \mathrm{Myr}$ for this object unless this object is extincted by circumstellar dust. At a distance of 59 pc, 2M1207A is considerably above the locus of the Pleiades, consistent with an age of $<10 \mathrm{Myr}$. 

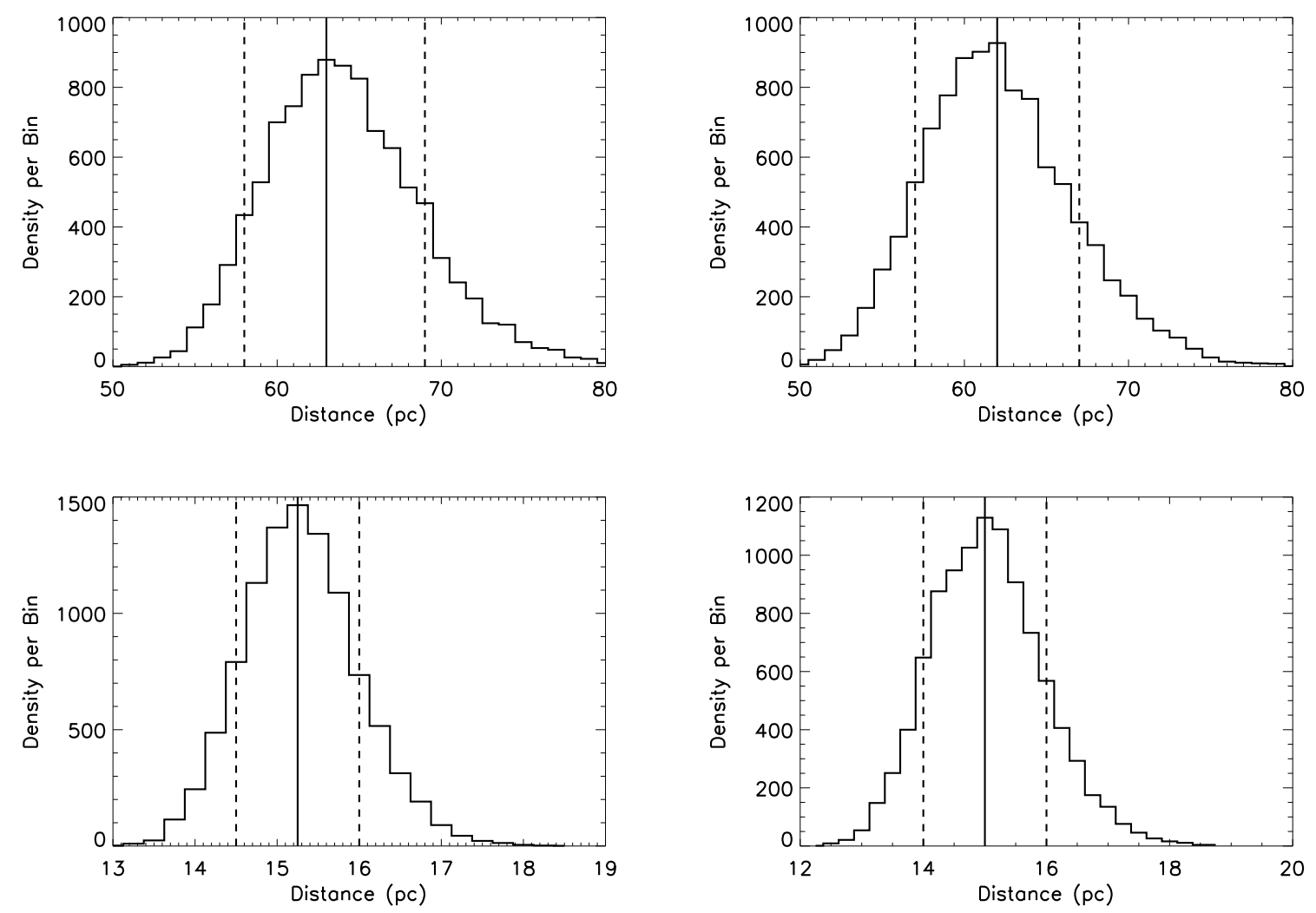

Fig. 2.- Histograms of the distributions of Monte-Carlo simulation observation fits before absolute parallax correction. Upper left: fits to the RA component of 2M1207. Upper right: fits to the DEC component of 2M1207. Lower left: fits to the RA component of LHS 2397a. Lower right: fits to the DEC component of LHS 2397a. The solid line marks the mean of each distribution and the dashed lines mark $1 \sigma$ from the mean. 

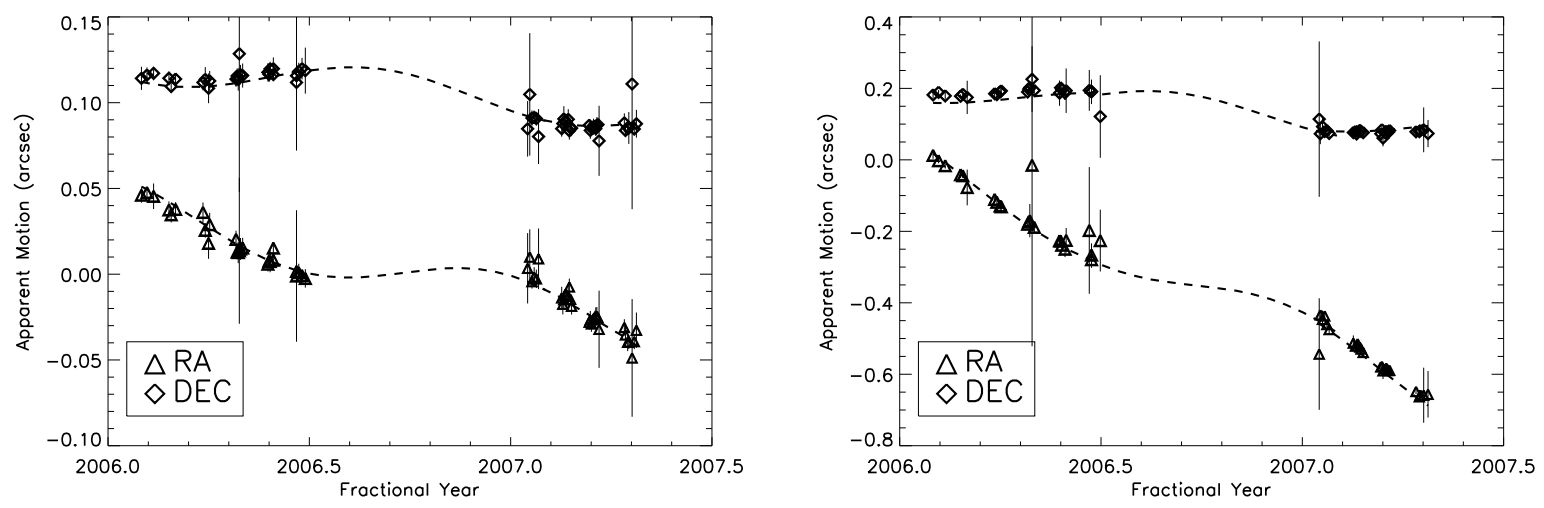

Fig. 3.- RA and DEC measured relative motion curves (before absolute parallax correction) for 2M1207A (left) and LHS 2397a (right). The dashed lines represent the 15.8 mas / 63 pc relative parallax best fit model for 2M1207A and the 66.7 mas / 15 pc relative parallax best fit model for LHS 2397a. Correcting for absolute parallax, we find an absolute parallax of $17.0_{-1.8}^{+2.3}$ mas and a best fit distance of $58.8 \pm 7.0 \mathrm{pc}$ (all $1.28 \sigma$ errors) for $2 \mathrm{M} 1207 \mathrm{~A}$ and an absolute parallax of $67.9_{-4.7}^{+5.3}$ mas (similar to the previous result of $62.6 \pm 4.0$ mas, Tinney 1996) and a best fit distance of $14.7 \pm 1.0$ pc for our standard LHS 2397a. 